\title{
Toll-like receptors as a potential immunotherapeutic target
}

\author{
Rania Zayed \\ Professor of Clinical and Chemical Pathology, Kasr Alainy, Cairo University
}

\begin{abstract}
Targeted or precision medicine has been gaining increasing interest in the past years as one of the major modalities of medical treatment. Targeted therapies are expected to be more effective than older forms of treatments being specific to certain patient populations who can benefit most without facing unacceptable side effects.

Immune system dysfunction is involved in diseases initiation and progression. Targeting the immune system to restore its ability to fight infection and disease is crucial.

Toll-like receptors (TLRs) are the fundamental component of the innate immune system and key regulators of acquired immunity. In humans, 10 TLRs proteins (TLR1-10) have been identified. Many polymorphisms in genes that encode TLRs and their signaling molecules have been associated with human disease progression and susceptibility. TLRs fulfill many of the criteria that are required to be considered potential therapeutic targets for the treatment of inflammation, cancer, infection, allergy and autoimmunity.
\end{abstract}

Keywords: TLR- targeted therapy-cancer-infection-autoimmunity 